Questions vives

\section{Questions Vives}

Recherches en éducation

$\mathrm{N}^{\circ} \mathbf{3 0} \mid \mathbf{2 0 1 8}$

Les effets formatifs du mémoire en formation initiale des enseignants

\title{
La place des inégalités sociales d'apprentissage comme objet et cadre d'analyse dans les mémoires de master
}

Comparaison des thèmes et références bibliographiques de

115 mémoires d'enseignants du primaire en formation initiale dans deux académies

Inequalities of learning as object and analysis framework in master dissertations in initial training of primary teachers. Comparison of themes and bibliographic references of 115 dissertations

\section{Claire Benveniste}

\section{CpenEdition}

\section{Journals}

\section{Édition électronique}

URL : https://journals.openedition.org/questionsvives/3164

DOI : 10.4000/questionsvives.3164

ISSN : $1775-433 \mathrm{X}$

Éditeur

Université Aix-Marseille (AMU)

Édition imprimée

Date de publication : 21 décembre 2018

ISBN : 978-2-912643-54-4

ISSN : $1635-4079$

Référence électronique

Claire Benveniste, «La place des inégalités sociales d'apprentissage comme objet et cadre d'analyse dans les mémoires de master ", Questions Vives [En ligne], № 30 | 2018, mis en ligne le 12 décembre 2018, consulté le 21 septembre 2021. URL : http://journals.openedition.org/questionsvives/3164 ; DOI : https://doi.org/10.4000/questionsvives.3164

Ce document a été généré automatiquement le 21 septembre 2021.

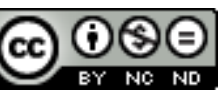

Questions Vives est mis à disposition selon les termes de la licence Creative Commons Attribution Pas d'Utilisation Commerciale - Pas de Modification 4.0 International. 


\section{La place des inégalités sociales d'apprentissage comme objet et cadre d'analyse dans les mémoires de master}

Comparaison des thèmes et références bibliographiques de 115 mémoires d'enseignants du primaire en formation initiale dans deux académies

Inequalities of learning as object and analysis framework in master dissertations in initial training of primary teachers. Comparison of themes and bibliographic references of 115 dissertations

\section{Claire Benveniste}

1 Les inégalités scolaires sont un objet de débat récurrent en France depuis les travaux sociologiques fondateurs des années 1960-1970, tant dans les sphères politiques et médiatiques qu'au sein des recherches en éducation. Les enseignants s'emparent-ils eux aussi de cette question des inégalités pour analyser les situations d'enseignement et d'apprentissage auxquelles ils sont confrontés? Quel peut être le rôle de la formation initiale des enseignants du primaire dans la construction d'une professionnalité réflexive qui leur permettrait de comprendre et peut-être dépasser leurs difficultés à faire apprendre une partie de leurs élèves, majoritairement ceux de milieux populaires? L'élaboration du mémoire de master MEEF en formation initiale permet-elle aux futurs enseignants de penser les conditions de possibilité d'une réduction des inégalités sociales d'apprentissage dans leurs classes? 


\section{Les inégalités sociales d'apprentissage comme cadre d'analyse des pratiques d'enseignement?}

2 Les enquêtes de «suivi de panels " des années 1970 s'intéressent principalement aux inégalités sociales de parcours, d'orientation et de diplômes. Elles montrent une élévation rapide du niveau de formation des nouvelles générations ${ }^{1}$ mais nous savons aujourd'hui que cette élévation des taux d'accès au baccalauréat et à l'enseignement supérieur n'a pas concerné tous les milieux sociaux de manière égale. Malgré les débats auxquels les analyses statistiques peuvent donner lieu (Rochex, 2013, p. 16), les chercheurs s'accordent pour affirmer que la « démocratisation qualitative » du système éducatif français n'a pas eu lieu (au sens de A. Prost, 2001) puisque le niveau de formation auquel s'opère une différenciation sociale des cursus et des trajectoires scolaires a simplement reculé (Rochex, 2013, p.14). Ces enquêtes des années 1970 peuvent donner lieu à des interprétations déterministes et défaitistes pour les enseignants en laissant dans l'ombre les acquisitions de savoirs et de compétences qui sont au cœur de leur travail. Depuis la fin des années 1980, des enquêtes statistiques nationales et internationales mesurent les «performances» des élèves en matière d'acquisitions de savoirs et de compétences. Malgré les controverses politiques, méthodologiques et théoriques qu'elles suscitent (Rochex, 2013 ; Vrignaud, 2006), ces enquêtes ont le mérite de montrer d'une part que la France est un des pays de l'OCDE où l'influence de l'origine sociale sur les performances des élèves est la plus importante, et d'autre part que la proportion des élèves dont les acquis sont les plus faibles s'accroît sensiblement ${ }^{2}$. Ces inégalités d'apprentissage sont présentes dès l'école primaire et peuvent conduire à de forts décrochages cognitifs sans entraver nécessairement la progression des élèves dans leur cursus scolaire, conduisant certains auteurs à parler de « décrochage de l'intérieur » (Bautier, Bonnéry, Terrail et al., 2002).

3 Pour dépasser ces constats d'impuissance, les approches statistiques sont complétées depuis les années 1990 par des travaux qui étudient les inégalités en train de se faire, au cœur des dispositifs, situations et pratiques d'enseignement. Ces recherches et leurs cadres théoriques - sur lesquels nous reviendrons par la suite-permettent de déconstruire les simplifications qui imputent la responsabilité des inégalités scolaires aux élèves eux-mêmes, aux manques de leurs milieux familiaux, ou à l'inverse au fonctionnement inéluctable d'une École reproductrice. Ces apports peuvent problématiser les situations vécues par les enseignants dans leurs salles de classe, leur permettant de mieux comprendre les processus de production des inégalités sociales d'apprentissage et les combattre plus efficacement, ou du moins ne pas les creuser, dans le quotidien des situations d'enseignement et d'apprentissage.

4 Nous souhaitons questionner la formation initiale des enseignants du primaire dans les ESPE $^{3}$ à l'aune de cet enjeu en nous focalisant sur l'élaboration du mémoire de master $\mathrm{MEEF}^{4}$. Ce dernier est considéré ici comme un dispositif de formation relevant à la fois du scientifique et du professionnel (Delarue-Breton, 2014), ouvrant un espace privilégié pour fonder l'analyse des pratiques sur la recherche en éducation. Sans tomber dans l'illusion d'une formation toute-puissante qui transformerait à coup sûr les représentations, les conceptions et les pratiques des enseignants ${ }^{5}$, nous nous demandons dans quelle mesure les mémoires de master MEEF premier degré sont l'occasion pour les enseignants stagiaires de changer de regard sur leurs pratiques d'enseignement en les analysant sous 
l'angle des inégalités sociales d'apprentissage. Cela impliquerait notamment de mobiliser dans les mémoires des travaux de recherche qui considèrent les pratiques et les situations scolaires comme socialement construites et situées et qui prennent en compte les dimensions sociales et contextuelles des difficultés d'apprentissage des élèves.

C'est pourquoi nous avons analysé les thèmes et les références bibliographiques de 115 mémoires soutenus en 2015 et 2016 dans deux ESPE dont les différences seront soulignées dans la discussion de nos résultats. Nos analyses montrent que les réflexions sociologiques sur les inégalités et la démocratisation de l'accès au savoir sont loin d'être centrales dans les mémoires des stagiaires, et ce dans les deux ESPE étudiées. Les contrastes dans certains résultats montrent également que la formation à ou par ${ }^{6}$ la recherche ne peut pas être considérée comme un « allant de soi ». Pour contextualiser ces résultats, nous analysons dans un premier temps la place des questions liées aux inégalités scolaires et la place du mémoire et de la recherche dans le discours officiel sur la formation des enseignants du primaire.

\section{Un discours officiel sur la formation des enseignants et la réduction des inégalités ?}

6 Trouver une cohérence officielle aux discours tenus sur la formation n'est pas aisé tant les variations académiques sont importantes dans la mise en place des directives nationales ${ }^{7}$. De plus, le discours politique médiatisé n'est pas toujours en cohérence avec les textes officiels écrits concernant les ambitions et les principes qui doivent sous-tendre la formation.

7 Au début des années 1990, on doute de l'efficacité du système éducatif français et de l'adéquation de la formation et des pratiques des enseignants aux nouveaux publics manifestant moins de connivence avec les savoirs et les attentes scolaires. À la création des IUFM en 1990, les discours politiques affirment que le caractère universitaire de la nouvelle formation doit permettre d'améliorer la pratique des maitres, dans un double souci de performance et de démocratisation du système éducatif (Gomez, 2001). Il en est de même lorsque le ministre Vincent Peillon affirme dans son discours de lancement des ESPE en juillet $2013^{8}$ que nos « performances éducatives sont inacceptables parce qu'elles s'accompagnent en plus d'un accroissement des inégalités ", ajoutant pour justifier la réforme de la formation que « le déterminant le plus net de cette réussite [des élèves] c'est l'engagement et la qualification des professionnels ». Bien qu'il ne soit jamais réellement défini (inégalités de parcours, d'orientation, de diplômes, d'acquisitions ?), cet objectif de réduction des inégalités sociales de réussite scolaire se situe au cœur de la communication politique à la création des ESPE en 2013.

8 En revanche, les textes officiels écrits cadrant la formation des enseignants entre 1989 et 2015 au niveau national sont loin d'être centrés sur ces questions. En 1989, le rapport Bancel insiste sur la définition d'objectifs d'enseignement prenant en compte les enjeux sociaux des savoirs scolaires (Bancel, 1989, p. 3), les obstacles et ruptures « inhérentes aux savoirs enseignés » (p.4) et l'identification des "besoins spécifiques » de chaque élève pour lui " apporter l'aide personnalisée dont il a besoin» (p. 4). Les pédagogies actives, par projet, par situations-problèmes sont préconisées dans le rapport $(p .4,6)$ mais sans évoquer leur caractère socialement situé, ces pédagogies pouvant être éloignées des modes de socialisation des classes populaires sur de nombreux points ${ }^{9}$. Le rapport indique 
tout de même que des «notions de psycho-sociologie et de sociologie de l'éducation sont indispensables» pour la gestion des apprentissages (p.6), mais il ne précise pas lesquelles.

Plus récemment, le référentiel des compétences professionnelles de 2007 préconise des " éléments de sociologie et de psychologie permettant de tenir compte de la diversité des élèves et de leurs cultures » (MEN, 2007a, p. 16) ; et le référentiel de 2013 indique pour la compétence P3 qu'il faut adapter «son enseignement et son action éducative à la diversité des élèves » en prenant en compte les « préalables et les représentations sociales (genre, origine ethnique, socio-économique et culturelle) pour traiter les difficultés éventuelles dans l'accès aux connaissances" (MEN, 2013a, p.5). Les propositions nationales d'enseignements pour le tronc commun de formation dans les ESPE mentionnent certes la "sociologie des publics", mais associée à la "gestion de la diversité » (MEN, 2013b, p. 11). Ces formulations hybrides témoignent de l'évolution des politiques éducatives depuis quelques décennies vers ce que J.-Y. Rochex nomme le «troisième âge des politiques d'éducation prioritaire " (Rochex, 2008) : les politiques de lutte contre les inégalités cèdent la place à des objectifs d'équité, de garantie du minimum, d'individualisation en fonction de "besoins éducatifs particuliers", de promotion de la « diversité » et de maximisation des potentiels et des chances de réussite de chacun.

\section{La place du mémoire et de la recherche dans la formation des enseignants du premier degré depuis 1989 : une évidence non consensuelle}

Entre 1989 et 2015, la formation initiale des enseignants s'universitarise et cherche à s'appuyer sur des savoirs formalisés issus de la recherche, ainsi que sur des dispositifs d'articulation "pratique-théorie-pratique» (Altet, 2000 ; Perrenoud, Altet et al., 2010). Renouvelant le "genre discursif du mémoire universitaire " (Delarue-Breton, 2014), le mémoire de master MEEF est emblématique de cette articulation entre les registres scientifique et professionnel souhaitée dès la création des IUFM en 1990 mais qui n'a pas été constamment soutenue au cours des différentes réformes de la formation. Le rapport Bancel introduit en 1989 «la rédaction d'un mémoire centré sur une question disciplinaire, didactique ou éducative [qui] constituera une première initiation à la formation à et par la recherche » (Bancel, 1989, p. 11). La place du mémoire professionnel est confirmée par une nouvelle circulaire en 2002 (le mémoire «représente l'aboutissement de la démarche réflexive développée au cours de la formation » mais il n'est alors plus question que d'« éclairages théoriques " ou d'« étayage documentaire ", MEN, 2002, p. $9^{10}$ ). En 2007, toute mention d'un mémoire professionnel disparaît dans la nouvelle circulaire (MEN, 2007b), avant que le processus de mastérisation de la formation en 2010 ne rende à nouveau timidement obligatoire la « production d'une recherche, sous forme de soutenance d'un mémoire par exemple » (MEN, 2009, p. 3).

11 À la création des ESPE, l'arrêté du 27 août 2013 est plus explicite et indique que «la formation s'appuie sur une activité d'initiation à la recherche » (MEN, 2013c, article 7). En 2015, une note rédigée par le ministère pour réduire la diversité des attendus pour le mémoire selon les académies définit le mémoire MEEF comme relevant à la fois du professionnel (le sujet de recherche doit être « en lien avec la pratique professionnelle ») 
et du scientifique puisque le travail de rédaction du mémoire « prend appui sur une revue de travaux qui alimente la construction d'une problématique, les choix méthodologiques et l'analyse des résultats [...] dans un va-et-vient entre données empiriques et confrontation à l'état des connaissances, une méthodologie de recueil de données, un recueil de corpus et une analyse » (MEN, 2015, p. 2). Malgré des variations dans les termes utilisés (" activité (autonome) de recherche », "formation à la recherche», «formation par la recherche ", " contenu de recherche ", " démarche de recherche », " expérience de recherche » et "initiation à la recherche»), ces textes nationaux portent aujourd'hui l'ambition d'une formation ou d'une initiation à la recherche qui doit permettre de «se tenir informé des acquis de la recherche afin de pouvoir s'engager dans des projets et des démarches d'innovation pédagogique visant à l'amélioration des pratiques »(p.1), mais aussi d'une formation par la recherche au sens où les stagiaires sont tenus de mener une réelle activité de recherche encadrée par des chercheurs ${ }^{11}$ qui peut « constituer un apport pour le champ de la recherche en éducation » (p. 3).

Malgré des variations synchroniques, la figure de l'enseignant qui traverse ce discours officiel est bien celle d'un praticien réflexif qui se sert du registre scientifique pour penser et améliorer sa pratique professionnelle afin de gagner en efficacité. Le référentiel des compétences professionnelles de 2013 indique que l'enseignant doit « réfléchir sur sa pratique - seul et entre pairs - et réinvestir les résultats de sa réflexion dans l'action ", «mettre à distance sa pratique pour se donner les moyens de l'améliorer » (MEN, 2013, compétence 14).

Nous proposons alors de ne pas considérer la réflexivité comme la capacité en général de prendre de la distance, quel que soit le problème, et de se demander avec B. Schneuwly (2015) : à quoi devrait réfléchir ce praticien réflexif? Au regard de quels enjeux et de quelles finalités doit-il mesurer « l'amélioration » et l'« efficacité » de ses pratiques ? Des pratiques «efficaces» contribuent-elles nécessairement à réduire les inégalités? En analysant les thèmes et les références bibliographiques de 115 mémoires provenant de deux ESPE, nous cherchons à savoir d'une part si les enseignants stagiaires se posent la question des inégalités lorsqu'ils sont tenus d'analyser leurs pratiques en élaborant leur mémoire, et d'autre part s'ils mobilisent les travaux de recherche qui mettent au jour les mécanismes de construction des inégalités d'apprentissage au sein des classes. Les parties suivantes détaillent tour à tour la composition de notre corpus, les résultats concernant les thèmes des mémoires puis la méthodologie, les résultats et la discussion de l'analyse des références bibliographiques.

\section{Description du corpus de 115 mémoires recueillis dans deux ESPE}

Le nombre de mémoires recueillis pour chaque ESPE (tableau 2) correspond au nombre de mémoires déposés par les formateurs sur la plateforme en ligne DUMAS ${ }^{12}$. La disproportion entre le nombre de mémoires recueillis dans l'ESPE $n^{\circ} 1$ (96 mémoires) et dans l'ESPE $n^{\circ} 2$ (19 mémoires) est prise en compte dans l'analyse statistique par la suite lorsque nous établissons des différences significatives entre les deux ESPE. Ce mode de recueil à distance via le dépôt en ligne des «bons » mémoires n'est certes pas aléatoire et représentatif de l'ensemble des mémoires de ces ESPE, mais il a l'avantage de nous fournir une image fidèle de ce qui est attendu par les institutions de formation qui valorisent ces 
mémoires en les déposant en ligne dès lors qu'ils obtiennent une note supérieure ou égale à $16 / 20$.

En 2015 et 2016, les deux ESPE de notre recueil n'ont pas les mêmes attendus formels pour le mémoire en fonction du statut des stagiaires, inscrits ou non en M2 MEEF (voir tableau 1). La lecture du tableau 1 permet de comprendre la composition de notre corpus (tableau 2) :

- Pour l'ESPE $\mathrm{n}^{\circ} 1$, nous avons recueilli à la fois les mémoires des PES-Master et les «écrits réflexifs » des FSTG (fonctionnaires stagiaires) car ils sont soumis aux mêmes exigences scientifiques et au même accompagnement même si les attendus formels diffèrent.

- Pour l'ESPE $n^{\circ} 2$, nous n'avons en revanche pas recueilli les productions des DU (Diplômes universitaires, créés en 2015-2016) car il s'agit d'un tout autre cursus avec un accompagnement et des attendus formels éloignés de ceux d'un mémoire.

Tableau 1 : Travaux attendus en fonction du parcours des stagiaires pour les mémoires soutenus en 2015 et 2016

\begin{tabular}{|l|l|l|}
\hline & $\begin{array}{l}\text { Stagiaires inscrits en M2 MEEF : } \\
\text { lauréats du concours provenant de } \\
\text { M1 MEEF ou de tout autre M1 }\end{array}$ & $\begin{array}{l}\text { Stagiaires non inscrits en M2 MEEF : lauréats } \\
\text { du concours déjà titulaires du master MEEF, } \\
\text { d'un autre master, ou dispensés de master }\end{array}$ \\
\hline $\begin{array}{l}\text { ESPE } \\
\mathbf{n}^{\mathbf{0}} \mathbf{1}\end{array}$ & $\begin{array}{l}\text { Mémoire MEEF : 12000 mots } \\
(30 \text { pages environ) }\end{array}$ & $\begin{array}{l}\text { Mémoire, écrit professionnel, écrit réflexif, } \\
\text { compris entre 6 000 et 10 000 mots (15-25 pages) }\end{array}$ \\
\hline $\begin{array}{l}\text { ESPE } \\
\mathbf{n}^{\mathbf{0}} \mathbf{2}\end{array}$ & $\begin{array}{l}\text { Mémoire MEEF : cinquantaine de } \\
\text { pages = 100 000 caractères environ } \\
\text { (espaces compris) }\end{array}$ & $\begin{array}{l}\text { Production écrite individuelle (10 pages) } \\
\text { Présentation orale collective. }\end{array}$ \\
\hline
\end{tabular}

Note: en gris, les travaux que nous n'avons pas inclus dans notre recueil

Tableau 2 : Taille et composition du corpus de mémoires recueillis sur la plateforme en ligne DUMAS

\begin{tabular}{|l|l|l|l|}
\hline & ESPE $^{\circ} \mathbf{1}$ & ESPE $^{\circ} \mathbf{2}$ & Total \\
\hline Nombre de mémoires & 96 & 19 & 115 \\
\hline Dont FSTG (étudiants titulaires d'un M2, en formation adaptée) & 40 & $/$ & \\
\hline Dont M2 & 56 & 19 & 78 \\
\hline Soutenus en juin 2015 & 30 & 10 & 40 \\
\hline Soutenus en juin 2016 & 66 & 9 & 75 \\
\hline Nombre de références bibliographiques & 1437 & 479 & 1916 \\
\hline
\end{tabular}




\section{Des thèmes de mémoires correspondant aux pratiques contemporaines d'enseignement, sans préoccupation apparente pour les inégalités}

Nous avons défini deux ensembles pour analyser les thèmes des mémoires à partir de leurs titres et de leurs résumés :

- Les thèmes ancrés dans une ou plusieurs discipline(s) scolaire(s): langage/écrit/oral, sciences, mathématiques, histoire-géographie/espace-temps, EPS, arts.

- Les thèmes transversaux qui correspondent pour notre recueil à la gestion de l'autorité/le climat scolaire/la violence, les relations école-famille, les inégalités sociales de réussite scolaire, les inégalités sexuées, les élèves allophones, la bienveillance, les élèves à besoins spécifiques, l'illettrisme, le travail d'équipe en établissement, la motivation, le travail de groupe, la coopération, la pédagogie institutionnelle, l'autonomie, les «éducation à », la pédagogie de projet, la démarche d'investigation/résolution de problème, la manipulation, le jeu, les TICE, la pédagogie Montessori, les ateliers philosophiques.

Ces deux ensembles peuvent être croisés pour caractériser les mémoires qui se centrent sur un thème transversal à partir d'apprentissages disciplinaires, ce qui explique que la somme des deux premières colonnes du tableau 3 dépasse $100 \%$. Nous avons par ailleurs caractérisé certains thèmes transversaux comme emblématiques des évolutions curriculaires contemporaines (dernière colonne du tableau 3 ) : autonomie, éducation à, pédagogie de projet, de la coopération, actives, ludiques, d'inspiration socioconstructiviste (résolution de problème, démarche d'investigation, travail de groupe...), pédagogies institutionnelles et pédagogie Montessori.

Tableau 3 : Analyse des thèmes des 115 mémoires : nombre (et \%) de mémoires centrés autour...

\begin{tabular}{|l|l|l|l|l|l|}
\hline $\begin{array}{l}\text { Nombre } \\
\text { (et \%) de } \\
\text { mémoires } \\
\text { centrés } \\
\text { autour... }\end{array}$ & $\begin{array}{l}\text { thème } \\
\text { transversal }\end{array}$ & $\begin{array}{l}\text {...d'une ou } \\
\text { plusieurs } \\
\text { discipline } \\
\text { (s) scolaire } \\
(\mathrm{s})\end{array}$ & $\begin{array}{l}\text {...d'un thème } \\
\text { uniquement } \\
\text { transversal et } \\
\text { non } \\
\text { disciplinaire }\end{array}$ & $\begin{array}{l}\text {...d'un thème } \\
\text { uniquement } \\
\text { disciplinaire et } \\
\text { non } \\
\text { transversal }\end{array}$ & $\begin{array}{l}\text {..d'un thème } \\
\text { emblématique des } \\
\text { évolutions } \\
\text { curriculaires } \\
\text { contemporaines }\end{array}$ \\
\hline $\begin{array}{l}\text { ESPE } \mathrm{n}^{\circ} 1 \\
(96 \\
\text { mémoires })\end{array}$ & $92(96 \%)$ & $53(55 \%)$ & $43(45 \%)$ & $4(4 \%)$ & $91(95 \%)$ \\
\hline $\begin{array}{l}\text { ESPE } \\
(19 \\
\text { mémoires })\end{array}$ & $19(100 \%)$ & $15(79 \%)$ & $4(21 \%)$ & 0 & $18(95 \%)$ \\
\hline
\end{tabular}

Les thèmes des 96 mémoires recueillis à l'ESPE $\mathrm{n}^{\circ} 1$ sont définis par les stagiaires parmi plusieurs domaines d'investigation non disciplinaires proposés dans le descriptif de l'UE "mémoire» de cette ESPE. La question des inégalités sociales de réussite scolaire, pourtant centrale dans les discours politiques qui entourent la mise en place des ESPE (voir précédemment), est absente de ces propositions, ce qui peut expliquer qu'un seul mémoire se centre sur les inégalités dans cette ESPE. Il en est de même dans l'ESPE $n^{\circ} 2$ 
(un seul mémoire centré sur les inégalités d'apprentissage) mais l'analyse des thèmes est moins pertinente dans cette ESPE car les mémoires sont principalement cadrés par les options de recherche thématiques dans lesquelles sont inscrits les stagiaires : les thèmes relevés sont donc conformes aux cinq options ayant déposé des mémoires sur la plateforme en ligne mais ne sont pas forcément représentatifs de l'ensemble des mémoires soutenus ${ }^{14}$.

Les 43 mémoires de l'ESPE $n^{\circ} 1$ centrés sur un thème transversal non lié à une discipline scolaire montrent que les stagiaires peuvent s'intéresser à des compétences transversales, par exemple à la gestion de l'autorité, au climat scolaire et à la violence (27 mémoires parmi les 43), à l'autonomie (9 mémoires) ou à la coopération dans la classe (8 mémoires), sans s'adosser à des apprentissages disciplinaires. Ces observations vont dans le sens des recherches qui mettent en évidence une invisibilisation des enjeux de savoirs en classe en lien avec le développement de ces compétences transversales dans les curricula (Bernstein, 2007 ; Coulange, 2011 ; Margolinas \& Laparra, 2011).

Dans les deux ESPE, $95 \%$ des mémoires analysent des pratiques professionnelles caractéristiques des nouvelles manières d'apprendre et de faire la classe : l'élaboration du mémoire participe donc de la diffusion dans les ESPE de ces pratiques aujourd'hui «dominantes». Or les recherches en éducation qui analysent les origines de ces pratiques, les modèles sociaux qui les sous-tendent et leurs effets socialement différenciés sur les apprentissages des élèves montrent que des choix pédagogiques ne sont jamais porteurs d'égalité $a$ priori dans l'accès au savoir mais que cela dépend de la manière dont ils sont mis en œuvre, quelles que soient les intentions initiales des enseignants. Même si les mémoires ne sont pas explicitement centrés sur les inégalités dans leurs titres et leurs résumés, nous nous demandons dans la suite de cette étude si les enseignants stagiaires analysent tout de même leurs pratiques, leurs difficultés et les difficultés de leurs élèves à la lumière des travaux sur les inégalités sociales d'apprentissage. Cela nécessite de revenir au préalable sur ces travaux et sur les cadres théoriques qui les sous-tendent afin de justifier les catégories retenues par la suite pour analyser les références bibliographiques des mémoires.

\section{Quels champs théoriques pour analyser les pratiques des enseignants caractéristiques de l'école contemporaine sous l'angle des inégalités d'apprentissage?}

De nombreux travaux de sociologie de l'école et des apprentissages s'attachent à objectiver les inégalités sociales de réussite scolaire et à rendre intelligibles les mécanismes de leur fabrication. L'enjeu est alors de ne pas considérer les pratiques d'enseignement et les situations scolaires comme neutres, naturellement efficaces ou égalitaires, mais bien comme des pratiques et des situations socialement situées et construites $^{15}$. Ces travaux contextualisent socio-historiquement les situations et les pratiques d'enseignement dans les évolutions curriculaires (Bautier \& Rayou, 2009 ; Richard-Bossez, 2015 ; van Brederode, 2016 ; Young, 2001) et dans les politiques éducatives contemporaines (Demeuse, Frandji et al., 2012). Les difficultés des élèves et la fabrication des inégalités sociales d'apprentissage sont considérées « comme résultant de la confrontation entre, d'une part, les dispositions socio-cognitives et socio-langagières 
des élèves, liées à leurs modes de socialisation, et qui les préparent de façon fort inégale à faire face aux réquisits des apprentissages scolaires et, d'autre part, l'opacité et le caractère implicite de ces réquisits, des modes de fonctionnement du système éducatif et [...] des pratiques professionnelles qui y sont mises en œuvre » (Bautier \& Goigoux, 2004).

Ces travaux sont inscrits dans le champ de la sociologie mais ils peuvent convoquer plusieurs champs théoriques. C'est le cas notamment de travaux socio-didactiques (Lahire \& Johsua, 1999 ; Losego, 2014 ; Margolinas, 2014), des approches qui mêlent la psychologie historique et culturelle de Vygotski aux autres sciences sociales (Joigneaux \& Rochex, 2008) mais aussi des recherches qui croisent l'ergonomie cognitive et la sociologie (Ria \& Rayou, 2008).

\section{Classification et analyse statistique des références présentes dans les bibliographies des mémoires}

\section{Précisions méthodologiques concernant nos catégories d'analyse}

Pour classer les 1916 références bibliographiques présentes dans les 115 mémoires de notre recueil (soit 1528 références uniques, certaines étant citées dans plusieurs mémoires), nous avons croisé plusieurs types de critères pour répondre à nos questions de recherche :

- Nous avons utilisé dans un premier temps les champs théoriques classiques tels que la sociologie de l'éducation, la psychologie des apprentissages, les didactiques, l'histoire de l'éducation, et l'analyse de l'activité et du travail (avec la didactique professionnelle). Ce découpage correspond aux références présentes dans les mémoires qui appartiennent toutes à l'une ou l'autre de ces disciplines de recherche.

- Il a fallu tenir compte également des objets de recherche pour la sociologie de l'éducation: sociologie du système scolaire (incluant par exemple les enquêtes quantitatives sur les trajectoires scolaires et les enquêtes plus qualitatives sur la construction sociale des diplômes), des inégalités d'apprentissages (travaux évoqués ci-dessus), de la famille, de l'enfance, du genre, de la culture.

- Les références de psychologie ont été catégorisées selon leur orientation théorique plus ou moins propice aux croisements avec des perspectives sociologiques sur les inégalités: psychologie historique et culturelle issue des travaux de Vygotski, psychologie clinique d'orientation psychanalytique, psychologie sociale, psychologie médicale liée à la rééducation et à la psychiatrie pédiatrique, psychologie cognitive et neurosciences.

- Pour les références de didactique, nous avons distingué la didactique liée à des objets disciplinaires et celle liée à des objets transversaux (comme les didactiques récentes de la coopération ou de l'évaluation). Nous avons également ajouté un critère pour les travaux didactiques basés sur des observations de classes qui peuvent éventuellement prendre en compte les dimensions sociales et contextuelles des situations d'enseignement et d'apprentissage (Bautier, 2010).

- Les références de recherche "hors éducation » ont été catégorisées en fonction de leur ancrage disciplinaire (littérature, histoire-géographie, mathématiques, sciences, médecine, linguistique, philosophie, psychologie, psychanalyse, sciences politiques) ${ }^{16}$.

En cherchant à catégoriser les références bibliographiques selon ces critères, nous avons constaté qu'un grand nombre d'entre elles ne correspondent pas à de la recherche en éducation. En nous inspirant d'autres études empiriques (Lhoste, 2005 ; Savaton, 2005), 
nous avons donc préalablement classé les références selon les distinctions suivantes: presse, dictionnaire, littérature jeunesse, ouvrages de révision pour le concours, instructions et rapports officiels, mais surtout distinction entre ouvrages ou articles de recherche (vulgarisés ou non, synthèses théoriques et/ou basés sur des données empiriques) et ouvrages que nous qualifions de "propositions de pratiques pédagogiques ». Ces derniers regroupent majoritairement des ouvrages pédagogiques destinés aux enseignants sous forme de pratiques à mettre en place plus ou moins " clés en main» ainsi que des manuels scolaires, des banques de séquences, des documents d'accompagnement ou ressources Éduscol. Lorsqu'un écrit nous semblait se soucier tout autant des fondements scientifiques que des propositions de pratiques pour la classe, nous avons regardé comment l'ouvrage est utilisé dans les mémoires concernés et quelles parties exactement sont citées pour le catégoriser du côté « recherche » ou « propositions de pratiques ».

Enfin, le nombre de références citées par mémoire variant beaucoup d'un mémoire et d'une ESPE à l'autre ( 15 en moyenne pour l'ESPE $n^{\circ} 1$ contre 25 pour l'ESPE $n^{\circ} 2$ ), nous avons mené les analyses statistiques au niveau des mémoires et non au niveau des références totales. Les résultats des tableaux 4 et 5 se lisent ainsi : «En moyenne, 36,3\% des références citées par un mémoire de l'ESPE $n^{\circ} 1$ sont des propositions de pratiques pédagogiques ».

\section{Des références issues de recherches en éducation dont la proportion varie considérablement selon les ESPE}

La proportion de références issues de la recherche en éducation varie du simple au double d'une ESPE à l'autre (tableau 4). Les mémoires de l'ESPE $n^{\circ} 1$ citent un peu plus de références de "propositions de pratiques" qui décrivent les situations vécues et les dispositifs mis en place sur le terrain que de références issues de la recherche en éducation qui peuvent analyser ces situations (36\% contre $29 \%$ ). Ce constat est accentué par le fait que les recherches en éducation citées par les mémoires de l'ESPE $n^{\circ} 1$ correspondent plus souvent à des ouvrages ou articles de synthèse théoriques qu'à des recherches basées sur des données empiriques pourtant plus propices aux rapprochements avec les situations professionnelles. C'est tout l'inverse pour les mémoires de l'ESPE ${ }^{\circ} 2$ qui ne citent que $7 \%$ d'ouvrages de "propositions de pratiques " contre $67 \%$ d'ouvrages de recherche en éducation et autant d'ouvrages "empiriques " que d'ouvrages de synthèse théorique. Ces différences sont accentuées par la proportion des références de recherches en éducation tirées d'ouvrages de vulgarisation qui a tendance à être plus élevée dans l'ESPE $n^{\circ} 1$.

Tableau 4 : Analyse des références bibliographiques des 115 mémoires (1/2)

\begin{tabular}{|l|l|l|l|}
\hline & ESPE no 1 & ESPE no $2^{\circ}$ & $\begin{array}{l}\text { Différence } \\
\text { significative ? }\end{array}$ \\
\hline $\begin{array}{l}\text { Propositions de pratiques } \\
\text { pédagogiques }\end{array}$ & $\begin{array}{l}36,3 \% \\
{[31,6-41,4]}\end{array}$ & $\begin{array}{l}7,3 \% \\
{[4,3-11,6]}\end{array}$ & oui $(\mathrm{p}<0,001)$ \\
\hline
\end{tabular}




\begin{tabular}{|l|l|l|l|}
\hline Recherches & $\begin{array}{l}35,8 \% \\
{[31,2-40,5]}\end{array}$ & $\begin{array}{l}78 \% \\
{[69,9-84,8]}\end{array}$ & oui $(\mathrm{p}<0,001)$ \\
\hline Recherches en éducation & $\begin{array}{l}29,1 \% \\
{[24,7-33,7]}\end{array}$ & $\begin{array}{l}67,1 \% \\
{[56,7-76,4]}\end{array}$ & oui (p<0,001) \\
\hline dont vulgarisation & $\begin{array}{l}25 \% \\
{[19,8-30,6]}\end{array}$ & $\begin{array}{l}16,9 \% \\
{[11,1-24]}\end{array}$ & $\begin{array}{l}\text { tendanciellement } \\
\text { significatif }\end{array}$ \\
\hline dont ouvrages/articles empiriques & $\begin{array}{l}21 \% \\
{[16,5-25,7]}\end{array}$ & $\begin{array}{l}31,8 \% \\
{[24,1-39,9]}\end{array}$ & oui (p<0,001) \\
\hline dont ouvrages/articles de synthèse & $\begin{array}{l}46,3 \% \\
{[16,5-25,7]}\end{array}$ & $\begin{array}{l}31,1 \% \\
{[24-38,6]}\end{array}$ & oui (p<0,001) \\
\hline Textes officiels & $\begin{array}{l}7,5 \% \\
{[5,4-9,8]}\end{array}$ & $\begin{array}{l}6,1 \% \\
{[3,3-10,4]}\end{array}$ & non \\
\hline $\begin{array}{l}\text { Autres (dictionnaires, rapports } \\
\text { littérature jeunesse...) }\end{array}$ & $\begin{array}{l}15,9 \% \\
{[12,3-19,7]}\end{array}$ & $\begin{array}{l}5,9 \% \\
{[3,2-10,3]}\end{array}$ & $/$ \\
\hline Références non codées ${ }^{17}$ & $3 \%[1,8-4,4]$ & $\begin{array}{l}0,9 \% \\
{[0,3-2,1]}\end{array}$ & $/$ \\
\hline
\end{tabular}

Note : pourcentage moyen et [intervalle de confiance à $95 \%$ ] estimés avec un modèle linéaire généralisé pour une distribution binomiale.

Lecture : en moyenne, 36,3\% des références citées par un mémoire de l'ESPE $n^{\circ} 1$ sont des propositions de pratiques pédagogiques.

En accord avec notre analyse des thèmes des mémoires, la très grande majorité $(85 \%)$ des ouvrages de "propositions de pratiques» concernent la mise en place de projets, de débats en classe, d'atelier-philo ou théâtre, de travail de groupe, d'activités ludiques, d'activités autonomes, de situations-problèmes, de démarches d'investigation, d'« éducation à » et d'activités proposées par des mouvements pédagogiques proches de l'Éducation nouvelle.

\section{Une analyse des pratiques professionnelles très peu menée sous l'angle des inégalités sociales d'apprentissage}

Nos résultats montrent sans ambiguïté que les mémoires analysés ne se centrent pas sur la question des inégalités sociales d'apprentissage (voir tableau 5), confirmant ainsi nos conclusions tirées de l'analyse des thèmes des mémoires. Seuls un tiers des mémoires de notre étude citent des références de recherche en sociologie de l'éducation et seuls un quart citent des travaux portant spécifiquement sur les inégalités sociales d'apprentissage (deux références seulement en moyenne dans ces mémoires). La sociologie des inégalités d'apprentissage représente environ $5 \%$ des références de recherches en éducation citées par les mémoires dans les deux ESPE. La didactique est très représentée dans les mémoires de l'ESPE $n^{\circ} 1$ ( $33 \%$ des références de recherches en éducation) mais seulement $10 \%$ de ces travaux didactiques reposent sur des observations de classe pouvant être socialement contextualisées. 
La psychologie est aussi mobilisée dans les deux ESPE (en moyenne, $33 \%$ des recherches en éducation citées dans les mémoires de l'ESPE $n^{\circ} 1$ appartiennent à la psychologie de l'éducation - soit 5 fois plus que la sociologie de l'éducation, et $14 \%$ dans l'ESPE ${ }^{\circ} 2$ ) mais il s'agit très majoritairement de la psychologie cognitive, de la psychologie génétique de Piaget, ou de références de neurosciences et neuro-éducation dans lesquelles le développement du sujet est peu pensé en interaction avec ses milieux et comme en apesanteur des rapports sociaux. Dans les deux ESPE, seuls 18 mémoires sur 115 citent des références de psychologie vygotskienne dans leur bibliographie et ils n'en citent alors qu'une ou deux en moyenne, ce qui explique les pourcentages très faibles obtenus pour ces travaux $(0,2 \%$ et $0,1 \%)$ parmi les références de psychologie.

Tableau 5 : Analyse des références bibliographiques des 115 mémoires (2/2)

\begin{tabular}{|c|c|c|c|}
\hline & ESPE $n^{\circ} 1$ & ESPE $n^{\circ} 2$ & $\begin{array}{l}\text { Différence } \\
\text { significative? }\end{array}$ \\
\hline $\begin{array}{l}\text { Recherches en éducation } \\
\text { parmi toutes les références }\end{array}$ & $\begin{array}{l}29,1 \% \\
{[24,7-33,7]}\end{array}$ & $\begin{array}{l}67,1 \% \\
{[56,7-76,4]}\end{array}$ & oui $(p<0,001)$ \\
\hline dont didactique disciplinaire & $\begin{array}{l}32,9 \% \\
{[23,4-43,2]}\end{array}$ & $\begin{array}{l}50,3 \% \\
{[30,8-69,3]}\end{array}$ & $\begin{array}{l}\text { tendanciellement } \\
\text { significatif }\end{array}$ \\
\hline dont sociologie & $\begin{array}{l}6,9 \% \\
{[3,4-11,6]}\end{array}$ & $\begin{array}{l}15 \% \\
{[6,4-29,1]}\end{array}$ & $\begin{array}{l}\text { tendanciellement } \\
\text { significatif }\end{array}$ \\
\hline $\begin{array}{l}\text { dont sociologie des inégalités } \\
\text { d'apprentissages }\end{array}$ & $4,3 \%[1,8-7,7]$ & $\begin{array}{l}4,8 \% \\
{[1,6-10,4]}\end{array}$ & non \\
\hline dont psychologie & $\begin{array}{l}33,4 \% \\
{[24,3-43,2]}\end{array}$ & $\begin{array}{l}14,3 \% \\
{[7-26,5]}\end{array}$ & oui $(p<0,001)$ \\
\hline \multicolumn{4}{|l|}{ Didactique } \\
\hline $\begin{array}{l}\text { dont résultats issus d'observations « en } \\
\text { situation de classe » }\end{array}$ & $\begin{array}{l}10,3 \% \\
{[5,6-16,1]}\end{array}$ & $\begin{array}{l}45,4 \% \\
{[32,8-56,5]}\end{array}$ & oui $(p<0,001)$ \\
\hline \multicolumn{4}{|l|}{ Sociologie } \\
\hline $\begin{array}{l}\text { dont sociologie des inégalités } \\
\text { d'apprentissages }\end{array}$ & $\begin{array}{l}71,9 \% \\
{[45,5-95]}\end{array}$ & $\begin{array}{l}41,1 \% \\
{[10,3-78,3]}\end{array}$ & $\begin{array}{l}\text { tendanciellement } \\
\text { significatif }\end{array}$ \\
\hline dont sociologie du système scolaire & $\begin{array}{l}19,3 \% \\
{[2,3-38,3]}\end{array}$ & $\begin{array}{l}13,7 \% \\
{[1,5-33,2]}\end{array}$ & non \\
\hline \multicolumn{4}{|l|}{ Psychologie } \\
\hline $\begin{array}{l}\text { dont psychologie cognitive, génétique, } \\
\text { neurosciences }\end{array}$ & $\begin{array}{l}64,6 \% \\
{[48,9-78,9]}\end{array}$ & $\begin{array}{l}84,2 \% \\
{[61,4-96,3]}\end{array}$ & non \\
\hline $\begin{array}{l}\text { dont psychologie historique et } \\
\text { culturelle (Vygotski) }\end{array}$ & $0,2 \%[0-8,1]$ & $0,1 \%[0-9]$ & non \\
\hline
\end{tabular}




\begin{tabular}{|l|l|l|l|}
\hline $\begin{array}{l}\text { dont psychologie sociale (motivation et } \\
\text { interaction) }\end{array}$ & $\begin{array}{l}16,3 \% \\
{[7,9-26,2]}\end{array}$ & $\begin{array}{l}20,3 \% \\
{[6,9-41]}\end{array}$ & non \\
\hline $\begin{array}{l}\text { dont psychologie clinique et } \\
\text { psychanalyse }\end{array}$ & $5,9 \%[0-19,7]$ & $0,2 \%[0-2,7]$ & non \\
\hline
\end{tabular}

Note: pourcentages moyens et [intervalle de confiance à $95 \%$ ] estimés avec un modèle linéaire généralisé pour une distribution binomiale.

Lecture: en moyenne, $6,9 \%$ des références de recherches en éducation citées par un mémoire de I'ESPE n $^{\circ} 1$ sont des références de sociologie de l'éducation.

\section{Pourquoi si peu d'attention portée aux inégalités sociales d'apprentissage et de réussite scolaire dans les mémoires des deux ESPE?}

Notre étude plus globale des maquettes de master MEEF dans le cadre d'une recherche doctorale en cours montre que les stagiaires sont très peu confrontés à la problématique des inégalités en formation initiale dans les ESPE, que ce soit dans les cours magistraux théoriques, dans des options en plus petits effectifs ou lors de retours réflexifs sur des séquences d'enseignement menées en classe. Leur possible intérêt pour cette problématique dans leur mémoire pourrait donc provenir plutôt de leur parcours avant leur entrée à l'ESPE (pour lequel nous n'avons aucune donnée dans notre étude) ou de l'encadrement du mémoire. Qui encadre donc les mémoires dans nos deux ESPE, et comment?

Pour l'ESPE $\mathrm{n}^{\circ} 1$, aucun des directeurs des mémoires de notre recueil ne mène de recherches au sein d'un laboratoire axé sur les inégalités (nous verrons ci-dessous qu'une majorité de ces directeurs ne sont pas impliqués dans des activités de recherche) et seuls deux chercheurs du collège de " philosophie-SHS » de l'ESPE travaillent sur les inégalités scolaires au moment du recueil. F. Baluteau a montré cependant que la nature des savoirs sociologiques, les attentes des formés et les contraintes institutionnelles rendent difficiles les usages du savoir sociologique en formation initiale des enseignants (Baluteau, 2002), nous gardant bien de conclure qu'une présence accrue de chercheurs travaillant sur les inégalités d'apprentissages changerait nécessairement les résultats obtenus dans cette ESPE.

Dans l'ESPE $n^{\circ} 2$, les enseignements « recherche » sont adossés dès 2013 à une vingtaine de laboratoires de recherche de l'université de tutelle de l'ESPE et des trois universités partenaires. Ils sont assurés très majoritairement par les enseignantschercheurs de ces laboratoires, pouvant être ou non formateurs à l'ESPE. Les mémoires sont fortement cadrés par les thématiques des options et cela peut expliquer la plus forte représentation de la sociologie de l'éducation parmi les recherches en éducation ( $15 \%$ contre $7 \%$ dans l'ESPE $n^{\circ} 1$ ) alors que la sociologie des inégalités d'apprentissage y est moins importante: certaines options qui ont déposé des mémoires sur la plateforme DUMAS reposent sur la sociologie du genre et des arts mais aucune n'est véritablement centrée sur les inégalités sociales de réussite scolaire (même si certains directeurs de mémoires mènent des recherches sur cette question). 

qui travaillent sur les processus de production des inégalités. Seules 2 options sur 31 sont explicitement centrées sur la question des inégalités sociales d'apprentissage et elles n'ont pas déposé de mémoires sur la plateforme DUMAS, ce qui peut expliquer la faible proportion de références à des travaux de recherche ou à des cadres théoriques liés aux inégalités sociales d'apprentissage dans les mémoires recueillis pour cette ESPE. L'option ayant déposé le plus de mémoires dans l'ESPE n 2 (10 mémoires sur 19) - centrée sur les pratiques dites innovantes - est tout de même adossée à un laboratoire de recherche axé sur les processus de production des inégalités mais cela ne semble pas suffisant pour que les stagiaires mobilisent ces travaux: ces dix mémoires citent en moyenne autant de sociologie des inégalités d'apprentissage que les neuf autres mémoires de cette ESPE ${ }^{18}$.

\section{Comment interpréter les résultats contrastés entre les deux ESPE concernant la proportion de références de recherches en éducation?}

Pour l'ESPE $n^{\circ} 1$, la moindre importance des références de recherches en éducation pourrait être due au fait que $55 \%$ des mémoires de notre recueil dans cette ESPE sont dirigés par des formateurs qui ne sont pas engagés par ailleurs dans des activités de recherche (contre $5 \%$ pour les mémoires de l'ESPE $n^{\circ} 2$ ). Nous avons testé statistiquement cette hypothèse mais les analyses ne montrent pas de différences significatives quant aux types de références selon que le directeur de mémoire est «chercheur» ou «non chercheur ». Une autre hypothèse serait liée à la composition du recueil dans cette ESPE qui comporte 40 mémoires de PES Hors-Master (FSTG) pour lesquels les exigences formelles sont moins importantes (voir tableau 1) mais nous n'avons pas trouvé de différences significatives dans nos résultats pour les mémoires des PES Master et HorsMaster de l'ESPE $n^{\circ} 1$.

La sociologie des enseignants stagiaires de nos deux ESPE pourrait-elle expliquer nos résultats contrastés ? L'ESPE $\mathrm{n}^{\circ} 2$ exige un mémoire de master MEEF plus conséquent que l'ESPE $\mathrm{n}^{\circ} 1$ alors même que son académie de rattachement traverse une crise de recrutement plus aiguë que le reste du territoire : le seuil d'admission au CRPE en 2015 est descendu bien en dessous de la moyenne, le taux de réussite au concours est supérieur à $60 \%$ et tous les postes n'ont pas été pourvus en 2014 et 2015 dans cette académie (MENDEPP, 2015, 2016). L'académie de l'ESPE $n^{\circ} 1$ voit en revanche tous ses postes pourvus en 2014 et 2015 et recrute ses enseignants stagiaires avec un seuil de 10/20 et avec un taux de réussite au concours de l'ordre de $40 \%$. Ces éléments nous laissent penser que les caractéristiques sociologiques des enseignants stagiaires de l'ESPE $n^{\circ} 2$ sont sensiblement différentes des tendances observées au niveau national allant dans le sens d'une élévation de l'origine des enseignants du premier degré sur l'échelle sociale (Farges, 2011, p. 171). Malgré l'élévation globale du niveau d'études des enseignants du premier degré (p. 164), les stagiaires recrutés dans l'ESPE $n^{\circ} 2$ avec de tels seuils d'admission et de tels taux de réussite ne sont pas nécessairement familiers des exigences littératiées requises pour rédiger un écrit long de recherche. Pourtant, ce sont les bibliographies de cette ESPE qui nous semblent refléter la plus grande adéquation avec les attendus du mémoire de master MEEF, la recherche en éducation y étant bien plus mobilisée que dans les mémoires de l'ESPE $n^{\circ} 1$.

Questions Vives, $N^{\circ} 30 \mid 2018$ 
Ce paradoxe s'explique avec nos données car en réalité, ces caractéristiques sociologiques des étudiants sont neutralisées dans notre étude puisque seuls les meilleurs mémoires satisfaisant les exigences du master MEEF et obtenant une note supérieure ou égale à 16/20 sont publiés sur la plateforme et ont donc été recueillis. Cela nous pousse finalement à chercher d'autres éléments qui pourraient différencier nos deux ESPE et à regarder de plus près les logiques d'organisation des enseignements liés à la recherche et à l'élaboration du mémoire.

\section{Des logiques d'organisation opposées pour les enseignements de recherche et d'accompagnement du mémoire dans les deux ESPE}

La proportion d'heures consacrées à l'accompagnement du mémoire dans l'ESPE $n^{\circ} 1$ est bien plus faible que dans l'ESPE $n^{\circ} 2$ ( $3 \%$ contre $22 \%$ de la maquette). L'ESPE $n^{\circ} 2$ organise les enseignements liés au mémoire sous forme d'options qui permettent d'aborder des questions disciplinaires, didactiques et professionnelles à partir d'appuis théoriques devant guider la réalisation du mémoire. Nous catégorisons donc ces options de recherche comme des enseignements liés à l'accompagnement du mémoire même si les 120 heures réparties sur les deux années de master n'y sont pas exclusivement consacrées. Le poids du mémoire dans la validation du master est également plus important dans l'ESPE $\mathrm{n}^{\circ} 2$ (tableau 6). Dans le contexte d'une année de stage extrêmement contrainte par un service de mi-temps en responsabilité en classe et un diplôme de master à valider, le faible poids et le faible accompagnement du mémoire dans

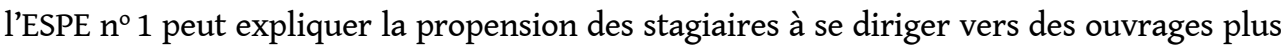
accessibles de propositions de pratiques pédagogiques.

Tableau 6 : Poids des enseignements liés à la recherche dans les deux ESPE du recueil

\begin{tabular}{|c|c|c|}
\hline & ESPE $n^{\circ} 1$ & ESPE $n^{\circ} 2$ \\
\hline $\begin{array}{l}\text { Nombre et proportion d'heures des UE } \\
\text { étiquetées " recherche" }\end{array}$ & $\begin{array}{l}10 \text { h sur } 346 \text { h au moment } \\
\text { du recueil }{ }^{19} \text {, } \\
\text { soit } 3 \% \text { de la maquette }\end{array}$ & 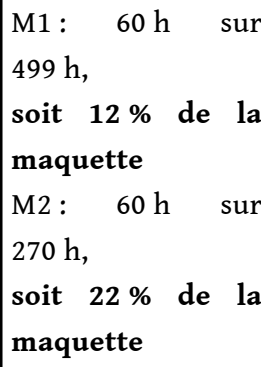 \\
\hline $\begin{array}{l}\text { Poids du mémoire et de la recherche dans } \\
\text { la validation du master }\end{array}$ & $\begin{array}{l}2014-2015: \\
10 \text { ECTS sur } 120(8 \%) \\
2015-2016: \\
20 \text { ECTS sur } 120(16 \%)\end{array}$ & $\begin{array}{l}34 \text { ECTS sur } 120 \\
\text { soit } \mathbf{2 8} \%\end{array}$ \\
\hline
\end{tabular}

Dans l'ESPE $n^{\circ} 1$, le texte de cadrage du mémoire en vigueur au moment de notre recueil précise que «l'ensemble des formateurs de l'ESPE intervenant dans la formation des étudiants et des professeurs stagiaires dans le cadre du master MEEF $1^{\text {er }}$ degré ont 
vocation à participer à la direction de mémoire ${ }^{20}$ ». Cette position est discutée lors d'une journée d'étude organisée dans cette ESPE en janvier 2017 par une commission de travail sur le mémoire. À l'inverse de ce qui est observé dans l'ESPE $n^{\circ} 2$ où les enseignantschercheurs s'impliquent quasiment exclusivement dans les enseignements liés au mémoire, les responsables de cette commission relèvent «le manque d'intérêt des enseignants-chercheurs des universités partenaires pour un mémoire à dimension professionnelle ${ }^{21} »$. Loin d'être purement organisationnelle et même si elle n'a pas d'influence directe sur les références citées dans les mémoires (voir précédemment), cette question de la direction des mémoires révèle donc le positionnement de l'ESPE $n^{\circ} 1$ qui semble valoriser la dimension professionnelle du mémoire au détriment de sa dimension scientifique.

39 Nous retrouvons pourtant dans les descriptifs et cadrages des deux ESPE les mêmes formules qui circulent entre les différents espaces institutionnels nationaux et académiques : "s'initier à la recherche ", " produire un travail personnel de recherche ", "à la lumière des résultats de la recherche", "favoriser l'analyse d'objets professionnels", "prendre le recul nécessaire par rapport à son expérience dans la classe », « développer une attitude réflexive », « distance critique par rapport à l'action », " aboutissement de la démarche de réflexion et d'analyse des pratiques »... Au-delà de ces formules, le discours institutionnel concernant l'élaboration du mémoire dans l'ESPE $n^{\circ} 1$ se caractérise par une certaine polyphonie: cohabitant avec des formules consacrant l'adossement à la recherche, d'autres éléments de ce discours font complètement disparaître le processus de formation par la recherche pour les enseignants du primaire (par exemple : «le mémoire de M2 MEEF ne vise pas à l'accroissement des connaissances dans la discipline, contrairement au mémoire de recherche disciplinaire $\left.{ }^{22} »\right)$. Ces formulations masquent néanmoins certaines tensions : la moitié des formateurs présents dans un atelier de travail sur le mémoire dans l'ESPE $n^{\circ} 1$ en 2017 affirme que «le mémoire de master MEEF constitue une recherche en éducation à proprement parler ", tandis que pour l'autre moitié des participants « la recherche en éducation est un support à l'élaboration d'un mémoire à finalité professionnelle ${ }^{23}$ ».

Dans l'ESPE $n^{\circ} 2$, la baisse horaire survenue en M1 entre 2016 et 2018 témoigne de contraintes et de tensions pesant sur ces enseignements liés au mémoire et à la recherche mais le document de présentation du master porte tout de même les ambitions d'un " approfondissement par la recherche »: les descriptifs des options de recherche et des attendus du mémoire insistent dans cette ESPE seulement sur la nécessité d'adosser la réflexion à des cadres conceptuels d'interprétation, à des ancrages théoriques et à des vaet-vient avec un recueil empirique mené sur le terrain de stage.

\section{Conclusion : quelle portée pour ces résultats ?}

41 Nous devons être prudent quant à la portée de nos analyses: des recherches plus qualitatives menées sur les usages des apports théoriques et concepts dans les mémoires des enseignants (Delarue-Breton \& Crinon, 2015 ; Ricard-Fersing, Crinon \& Birglin, 2001) montrent que la présence de références dans les bibliographies ne garantit en rien que les concepts et résultats de recherche soient travaillés et appropriés par les enseignants stagiaires $^{24}$. La focalisation de cette étude sur l'analyse quantitative des références bibliographiques laisse dans l'ombre des aspects qualitatifs qui permettraient d'une part d'analyser plus finement la manière dont les stagiaires se saisissent de la formation par la 
recherche quand elle est mise en œuvre, et d'autre part de donner la parole à des acteurs de la formation pour comprendre, relativiser et donner vie à des constats qui peuvent paraître abrupts.

Mais au-delà de nos résultats chiffrés, nous avons essayé de mener une analyse des dispositifs liés à l'élaboration du mémoire MEEF susceptibles de professionnaliser les futurs enseignants à une dimension particulière de leur métier: la réduction des inégalités sociales d'apprentissage. Nous montrons finalement que les travaux et les cadres théoriques qui permettent d'appréhender les inégalités sociales d'apprentissage sont très peu mobilisés dans les mémoires MEEF de notre recueil. Cela témoigne selon nous de l'absence de retour critique sur le caractère socialement situé - et donc potentiellement socialement différenciateur - des nouvelles manières d'apprendre et de faire la classe. Cela se vérifie dans le cas où l'institution de formation dit privilégier une formation à la recherche que les stagiaires mènent de manière autonome en élaborant leur mémoire avec très peu d'encadrement (ESPE $\left.n^{\circ} 1\right)$, mais aussi lorsqu'une formation par la recherche est mise en œuvre de manière ambitieuse malgré certaines tensions (ESPE $\left.n^{\circ} 2\right)$.

Avec les limites importantes que nous avons soulevées dans les deux ESPE de notre étude, le mémoire MEEF reste-t-il un dispositif de formation initiale susceptible de fonder l'analyse des pratiques sur la recherche en éducation? Est-il à même de porter les ambitions qui sous-tendent la formation des enseignants en ce qui concerne la lutte contre les inégalités sociales à l'école? Nos analyses permettent d'en douter mais les importantes variations académiques que nous avons mises au jour laissent peut-être imaginer d'autres possibles, avec l'espoir optimiste de voir la formation initiale des enseignants évoluer vers une temporalité moins contrainte.

\section{Textes et rapports officiels cités}

IGA-IGAENR (2016). La mise en place des écoles supérieures du professorat et de l'éducation au cours de l'année 2015-2016. Rapport n²016-062, septembre 2016.

MEN (2002). Principes et modalités d'organisation de la deuxième année de formation des enseignants et des conseillers principaux d'éducation stagiaires (plan de rénovation de la formation des enseignants du 27 février 2001). Circulaire $n^{\circ} 2002-070$ du 4 avril 2002 (encart BO $n^{\circ} 15$ du 11 avril 2002).

MEN (2007a). Cahier des charges de la formation des maîtres en IUFM. BO nº 1 du 4 janvier 2007.

MEN (2007b). Mise en œuvre du cahier des charges de la formation des maîtres. Circulaire $n^{\circ} 2007-045$ du 23 février 2007.

MEN (2009). Mise en place des diplômes nationaux de master ouverts aux étudiants se destinant aux métiers de l'enseignement dès la rentrée universitaire 2010. Circulaire $n^{\circ} 2009-0753$ du 23 décembre 2009. 
MEN (2013a). Référentiel des compétences professionnelles des métiers du professorat et de l'éducation, arrêté du $1^{\text {er }}$ juillet 2013.

MEN (2013b). Dossier du séminaire de lancement des ESPE, $1^{\mathrm{er}}$ juillet 2013. Repéré à http:// cache.media.education.gouv.fr/file/14/95/5/dossier_ESPE_259573_259955.pdf

MEN (2013c). Cadre national des formations dispensées au sein des masters « métiers de l'enseignement, de l'éducation et de la formation ». Arrêté du 27 août 2013, JO du 29 août 2013.

MEN (2015). Mise en œuvre du mémoire dans le cadre de la formation aux métiers de l'enseignement, de l'éducation et de la formation. Note $\mathrm{n}^{\circ}$ 2015-0459.

\section{Bibliographie}

Altet, M. (2000). L'analyse de pratiques : une démarche de formation professionnalisante? Recherche \& Formation, 35, 25-41.

Baluteau, F. (2002). Les usages du savoir sociologique dans un contexte de formation. Le cas des formateurs d'IUFM. Éducation et sociétés, 1, 73-85.

Bancel, D. (1989). Créer une nouvelle dynamique de la formation des maîtres. Paris : La Documentation française.

Bautier, É., Bonnéry, S., Terrail, J.-P., Bebi, A., Lesort, B. \& Branca-Rosoff, S. (2002). Décrochage scolaire : genèse et logique des parcours. Rapport de recherche pour la DPD/MEN.

Bautier, É. \& Goigoux, R. (2004). Difficultés d'apprentissage, processus de secondarisation et pratiques enseignantes : une hypothèse relationnelle. Revue française de pédagogie, 148, 89-100.

Bautier, É. \& Rayou, P. (2009). Les inégalités d'apprentissage : programmes, pratiques et malentendus scolaires. Paris : PUF.

Bautier, É. (2010). Recherche et différenciations socio-scolaires. Pratiques. Linguistique, littérature, didactique, 145-146, 17-21.

Bernstein, B. (2007). Classes et pédagogie : visible et invisible. Dans J.-P. Terrail \& J. Deauvieau (dir.), Les sociologues, l'école et la transmission des savoirs. Paris : La Dispute.

Coulange, L. (2011). Quand les savoirs mathématiques à enseigner deviennent incidents. Dans J.Y. Rochex \& J. Crinon (dir.), La construction des inégalités scolaires. Au cœur des pratiques et des dispositifs d'enseignement (pp. 33-44). Rennes : Presses universitaires de Rennes.

Crahay, M. (1989). Contraintes de situation et interactions maître-élève. Changer sa façon d'enseigner, est-ce possible? (Note de synthèse). Revue française de pédagogie, 88, 67-94.

Crahay, M., Wanlin, P., Issaieva, E. \& Laduron, I. (2010). Fonctions, structuration et évolution des croyances (et connaissances) des enseignants (Note de synthèse). Revue française de pédagogie, 172, 85-129.

Delarue-Breton, C. (2014). Le mémoire de master MEEF : un nouveau genre universitaire. Diversité, 177, 50-55.

Delarue-Breton, C. \& Crinon, J. (2015). De l'usage des concepts dans les mémoires de master des futurs enseignants. Le français aujourd'hui, 188, 79-88.

Demeuse, M., Frandji, D., Greger, D. \& Rochex, J.-Y. (dir.) (2012). Les politiques d'éducation prioritaire en Europe. Tome II. Quel devenir pour l'égalité scolaire? Lyon : ENS Éditions. 
Durler, H. (2014). Les pratiques du gouvernement de soi à l'école : les dispositifs pédagogiques de l'autonomie et leurs contradictions. Recherches en éducation, 20, 76-86.

Farges, G. (2011). Le statut social des enseignants français. Revue européenne des sciences sociales, 49 (1), 157-178.

Gomez, F. (2001). Le mémoire professionnel : objet de recherche et outil de formation. Bruxelles : De Boeck Supérieur.

Joigneaux, C. \& Rochex, J.-Y. (2008). La construction de l'élève à l'école maternelle : regards croisés et apports de Vygotski, Bernstein et Goody. Dans M. Brossard \& J. Fijalkow (dir.), Vygotski et les recherches en éducation et en didactiques (pp. 73-87). Bordeaux : Presses universitaires de Bordeaux.

Lahire, B. (1993). Culture écrite et inégalités scolaires. Sociologie de "l'échec scolaire » à l'école primaire. Lyon : Presses universitaires de Lyon.

Lahire, B. \& Johsua, S. (1999). Pour une didactique sociologique. Éducation et sociétés, 4(2), 29-56.

Lhoste, Y. (2005). Analyse de 24 mémoires professionnels sur 4 ans en SVT en formation initiale des enseignants $1^{\text {er }}$ et $2^{\text {nd }}$ degré. Dans M. Bailleul (dir.), Enseignants, formateurs et recherche $(s)$ en IUFM (Tome 2 - Formation et recherche(s)). Paris : L'Harmattan.

Losego, P. (2014). Rapprocher la sociologie et les didactiques. Revue française de pédagogie, 188, 5-12.

Margolinas, C. \& Laparra, M. (2011). Des savoirs transparents dans le travail des professeurs à l'école primaire. Dans J.-Y. Rochex \& J. Crinon (dir.), La construction des inégalités scolaires (pp. 19-32). Rennes : Presses universitaires de Rennes.

Margolinas, C. (2014). Connaissance et savoir. Concepts didactiques et perspectives sociologiques? Revue française de pédagogie, 188(3), 13-22.

MEN-DEPP (2015). Concours de professeurs des écoles dans l'enseignement public : deux fois plus de recrutements externes en 2014. Note d'information $n^{\circ} 21$, juin 2015.

MEN-DEPP (2016). Concours de professeurs des écoles dans l'enseignement public : $40 \%$ de recrutements externes en plus en 2015 . Note d'information $n^{\circ} 15$, mai 2016.

Perrenoud, P. (1985). Les pédagogies nouvelles sont-elles élitaires ? Dans La pédagogie à l'école des différences (pp. 105-118). Paris : ESF.

Perrenoud, P., Altet, M., Lessard, C. \& Paquay, L. (dir.) (2010). Conflits de savoirs en formation des enseignants. Paris : De Boeck.

Prost, A. (2001). La démocratisation de l'enseignement en France depuis la Seconde Guerre mondiale. Revue suisse des sciences de l'éducation, 23(1), 73-92.

Ria, L. \& Rayou, P. (2008). Sociologie et ergonomie cognitive au miroir des situations éducatives : le cas de l'entrée dans le métier des enseignants du $2^{\text {nd }}$ degré. Recherches \& éducations, 1, 105-119.

Ricard-Fersing, É., Crinon, J., \& Birglin, M.-J. (2001). Theory and its status in the professional dissertations by teachers. Dans C. Day \& D. van Veen (dir.), Educational Research in Europe, Yearbook (pp. 139-148). Anvers : Garant-Uitgevers.

Richard-Bossez, A. (2015). La construction sociale et cognitive des savoirs à l'école maternelle : entre processus différenciateurs et moments de démocratisation. Le cas des activités relatives à l'écrit en grande section. (Thèse de doctorat en sociologie). Université d'Aix-Marseille. 
Rochex, J.-Y. (2008). Les politiques d'éducation prioritaire en Europe, d'un « âge » et d'un pays à l'autre. Dans M. Demeuse, D. Frandji \& D. Greger (dir.), Les politiques d'éducation prioritaire en Europe (Tome 1) (pp. 409-451). Lyon : ENS Éditions.

Rochex, J.-Y. \& Crinon, J. (2011). La construction des inégalités scolaires : au cour des pratiques et des dispositifs d'enseignement. Rennes : Presses universitaires de Rennes.

Rochex, J.-Y. (2013). Des inégalités scolaires, des moyens de les mesurer et d'en étudier les processus de production. Le français aujourd'hui, 183(4), 9-28.

Savaton, P. (2005). La bibliographie du mémoire professionnel : pour quoi faire ? Dans M. Bailleul (dir.), Enseignants, formateurs et recherche(s) en IUFM (Tome 2 - Formation et recherche(s)). Paris :

L'Harmattan.

Schneuwly, B. (2015). À quoi réfléchit le praticien réflexif ? Le français aujourd'hui, 188, 29-38.

van Brederode, M. (2016). Savoirs scientifiques, malentendus et inégalités sociales à l'école. Les formes disciplinaires des SVT en $6^{e}$. (Thèse de Doctorat). Université Paris 8 Saint-Denis.

Vrignaud, P. (2006). La mesure de la littéracie dans PISA : la méthodologie est la réponse, mais quelle était la question? Revue française de pédagogie, 157, 27-41.

Young, M. (2001). Du « curriculum en tant que construction sociale » à la « spécialisation intégrative ». Quelques réflexions sur la sociologie du curriculum au Royaume-Uni (1971-1999). Revue française de pédagogie, 135, 29-34.

\section{NOTES}

1. D'abord au collège avec la première « explosion scolaire » dans les années 1960, puis au lycée avec la seconde « explosion scolaire » au milieu des années 1980.

2. Voir les résultats PISA 2015 pour la France: https://www.oecd.org/pisa/PISA-2015-FranceFRA.pdf.

3. ESPE : École supérieure du professorat et de l'éducation.

4. MEEF : Métiers de l'enseignement, de l'éducation et de la formation.

5. La lecture des travaux de psychologie sociale sur l'évolution des représentations, des croyances, des conceptions et des pratiques des enseignants empêche de tomber dans cet écueil (Crahay, 1989, 2010).

6. Nous gardons pour l'instant la juxtaposition des deux formules, ne pouvant trancher la question avant d'avoir présenté nos analyses.

7. En 2015-2016, la mission d'évaluation de la mise en place des ESPE indique par exemple qu'elle "a été frappée cette année encore par la diversité des situations rencontrées " (IGA-IGAENR, 2016, p. 5).

8. Archive consultée le 12 décembre 2017 à l'adresse: https://www.dailymotion.com/video/ x11hgca.

9. Perrenoud (1985) avait déjà synthétisé ces arguments dans un texte intitulé « Les pédagogies nouvelles sont-elles élitaires?».

10. La pagination correspond au document téléchargé depuis le site http:// www.education.gouv.fr/bo/2002/15/encart.htm.

11. Nous verrons par la suite que cette recommandation nationale ne s'applique pas toujours localement tant pour des questions d'effectifs (trop peu d'enseignants-chercheurs pour encadrer tous les mémoires) que de conception de la formation.

12. https://dumas.ccsd.cnrs.fr/, consulté en mai 2017. 
13. Par exemple, «Le développement de la pensée autonome à la maternelle ", «Comment la motivation des élèves peut permettre de franchir les obstacles à l'enseignement du secourisme en cycle 1 ?».

14. Les cinq options ayant déposé des mémoires en ligne sont: apprentissages mathématiques (approche didactique), la polyvalence (pluridisciplinarité et pratiques innovantes), activités physiques et nouvelles problématiques éducatives, musée (histoire des sciences et médiation), enseigner au prisme du genre.

15. Les travaux adoptant cette approche sont trop nombreux pour être cités ici mais nous pouvons citer à titre d'exemple les recherches de H. Durler (2014) sur l'autonomie ou de B. Lahire (1993) sur la culture écrite.

16. Ces résultats ne seront pas détaillés car ils ne sont pas utiles pour répondre à notre problématique.

17. Quelques références n'ont pas pu être codées quand le document original n'a pu être retrouvé, c'est souvent le cas de sites Internet dont l'URL n'existe plus.

18. Ces moyennes cachent des disparités entre les mémoires de cette option qui citent zéro, une ou deux références sur les inégalités d'apprentissage sauf un mémoire qui en cite six.

19. Les maquettes ont été modifiées entre 2014-2015 et 2015-2016 à l'ESPE $n^{\circ} 1$ mais de manière minime et difficilement mesurable car les maquettes habilitées ne correspondent pas toujours aux maquettes mises en œuvre sur le terrain.

20. Cadrage du mémoire de l'ESPE $\mathrm{n}^{\circ} 1$ obtenu dans le cadre d'un travail doctoral plus global sur la formation initiale.

21. Citations du compte rendu de la journée d'étude obtenu dans le cadre d'un travail doctoral plus global sur la formation initiale.

22. Cadrage du mémoire de l'ESPE $\mathrm{n}^{\circ} 1$ obtenu dans le cadre d'un travail doctoral plus global sur la formation initiale.

23. Citations du compte rendu de l'atelier obtenu dans le cadre d'un travail doctoral plus global sur la formation initiale.

24. Sans systématiser encore ces analyses complémentaires, nous avons observé par exemple que les stagiaires peuvent citer certains travaux qui étudient les effets des pédagogies actives sur les apprentissages des élèves de milieux populaires pour décrire ces pédagogies et "prouver » leur bien-fondé tout en "oubliant» d'évoquer la question sociologique soulevée initialement par l'auteur.

\section{RÉSUMÉS}

En se focalisant sur les thèmes et les bibliographies de mémoires de master MEEF, cet article interroge la formation initiale des enseignants du primaire à l'aune des enjeux de réduction des inégalités sociales à l'école. Nous analysons dans un premier temps la place des questions liées aux inégalités sociales de réussite scolaires dans le discours officiel sur la formation, ainsi que la place du mémoire et de la formation à et/ou par la recherche dans les cadrages de la formation initiale. La mise en œuvre de ces orientations nationales dans deux ESPE est ensuite étudiée via l'analyse statistique des thèmes et bibliographies de 115 mémoires MEEF premier degré. Les résultats montrent d'une part que les enseignants stagiaires privilégient plutôt des approches didactiques et psychologiques peu croisées à un questionnement sociologique sur la réduction 
des inégalités, et d'autre part que la formation à et/ou par la recherche ne va pas de soi malgré la mise en place de dispositifs qui s'en réclament.

By focusing on the themes and bibliographies of master MEEF dissertations, this article examines the initial training of primary school teachers in terms of reducing social inequalities at school. We first analyze the place of issues related to school inequalities in the official discourse on the training and the place of research during the initial training. The implementation of these national guidelines in two ESPE is then studied via the statistical analysis of the themes and bibliographies of 115 MEEF first-degree dissertations. The results show on the one hand that trainee teachers prefer rather didactic and psychological approaches, little crossed to a sociological questioning on the reduction of inequalities, and on the other hand, training for or through research is not self-evident despite the introduction of devices that claim to do so.

\section{INDEX}

Keywords : teacher training, MEEF master dissertations, social inequalities of learning, teacher's knowledge, bibliographical references

Mots-clés : formation des enseignants, mémoire de master MEEF, inégalités sociales

d'apprentissage, savoirs des enseignants, références bibliographiques

\section{AUTEUR}

\section{CLAIRE BENVENISTE}

Doctorante en sciences de l'éducation, Université Paris VIII, Laboratoire CIRCEFT-Escol (Centre interdisciplinaire de recherches sur la culture, l'éducation, la formation, le travail ; équipe Éducation Scolarisation). 